\title{
Magnetic Properties of a Novel $\mathrm{CeCo}_{0.715} \mathrm{Si}_{2.285}$ Compound
}

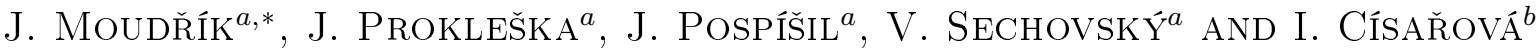 \\ ${ }^{a}$ Charles University in Prague, Faculty of Mathematics and Physics, Department of Condensed Matter Physics, \\ Ke Karlovu 5, 12116 Prague 2, Czech Republic \\ ${ }^{b}$ Charles University in Prague, Faculty of Science, Department of Inorganic Chemistry, \\ Hlavova 2030/8, 12843 Prague 2, Czech Republic
}

\begin{abstract}
We report on the basic physical properties of a novel $\mathrm{CeCo}_{0.715} \mathrm{Si}_{2.285}$ compound, mainly its rich magnetic phase diagram. The compound crystallizes in the $I-4 m 2$ space group structure with extremely elongated unit cell $(a=4.12 \AA, c=32.84 \AA)$. In a zero magnetic field it orders antiferromagnetically at $T_{\mathrm{N}}=10.5 \mathrm{~K}$ with another order-to-order transition at $9.5 \mathrm{~K}$. Under application of a magnetic field along the $c$-axis it manifests numerous magnetic transitions in small fields $(B<500 \mathrm{mT})$, resembling the so-called "devil's staircase" systems. Above $1 \mathrm{~T}$ the magnetization is almost constant up to $14 \mathrm{~T}$ (maximum magnetic field applied within our study) but considerably reduced $\left(0.3 \mu_{\mathrm{B}} / \mathrm{Ce}\right)$ with respect to the free $\mathrm{Ce}^{3+}$ ion. After removing the applied field, however, the high field state remains unchanged to be removed in negative fields. The compound also exhibits strong hysteresis of magnetization with respect to varying temperature or magnetic field. For fields applied along the $a$-axis typical behavior for the hard axis in the material with uniaxial anisotropy is observed.
\end{abstract}

DOI: 10.12693/APhysPolA.127.561

PACS: $75.30 .-\mathrm{m}, 75.30 . \mathrm{Gw}, 75.30 . \mathrm{Kz}$

\section{Introduction}

Cerium cobalt silicides attract long ongoing attention due to the richness of different ground states and their rather easy tuning by external (field, pressure) and internal (alloying, hydrogenation) parameters. The ground states of up-to-date known compounds range from a 'simple' antiferromagnet (CeCoSi $(P 4 / n m m)$, however, having a spin-density-wave gap under pressure, and indications of a magnetic quantum critical point [1]), intermediate valence/weak ferromagnetism in $\mathrm{CeCo}_{9+\delta} \mathrm{Si}_{4-\delta}$ system (I4/ $\mathrm{mcm},[2])$ to Pauli paramagnet behavior $\left(\mathrm{CeCoSi}_{2}(\mathrm{Cmcm})\right.$ [3]), Kondo paramagnetic behavior $\left(\mathrm{Ce}_{2} \mathrm{Co}_{0.8} \mathrm{Si}_{3.2}(\mathrm{P} 6 / \mathrm{mmm})[4]\right)$ and an alleged superconductor $\left(\mathrm{CeCoSi}_{3}\left(I_{4} \mathrm{~mm}\right)\right.$ [5]). In this paper we report on the preparation and characterization of the novel compound $\mathrm{CeCo}_{0.715} \mathrm{Si}_{2.285}$ and its magnetic properties.

\section{Experimental details}

The single crystal of $\mathrm{CeCo}_{0.715} \mathrm{Si}_{2.285}$ has been grown by a modified Czochralski pulling method in a triarc furnace under a high-purity argon atmosphere $(6 \mathrm{~N})$. A pulling speed of $6 \mathrm{~mm} / \mathrm{h}$ has been used. The growth was carried out from an off-stoichiometric composition of $\mathrm{Ce}: \mathrm{Co}: \mathrm{Si}=1: 1: 3.5$ of high-purity elements $(3 \mathrm{~N} \mathrm{Ce}$, further purified by solid state electrotransport [6], 4N5 Co and $6 \mathrm{~N} \mathrm{Si}$ ).

The obtained ingot has been wrapped in a tantalum foil $(3 \mathrm{~N})$, sealed in a quartz tube and annealed at $900^{\circ} \mathrm{C}$

*corresponding author; e-mail: jan.moudrik@gmail.com for one week under vacuum of $10^{-6}$ mbar. The single crystal has been oriented by Laue method and cut up to samples of a suitable shape with a fine wire saw. Specific heat and resistivity measurements were carried out using a commercial physical property measurement system (PPMS) while magnetization measurements were performed in a magnetic property measurement system (MPMS), both from Quantum Design, Inc.

\section{Results and discussion}

The tetragonal structure (spacegroup 119, I-4m2) of the studied compound was determined from single crystal diffraction measurements (Mo $\mathrm{K}_{\alpha}, 517$ reflections, 305 independent). It is noteworthy that the unit cell is extremely elongated along [001] direction $(a=4.12 \AA$, $c=32.84 \AA$ ). The structure determined from the single crystal diffraction is in agreement with powder diffraction data as well. The quality of the crystal was checked by Laue X-Ray diffraction, the phase purity was confirmed by a measurement with a scanning electron microscope equipped with an EDX spectroscopy option.

Temperature dependence of the specific heat of the $\mathrm{CeCo}_{0.715} \mathrm{Si}_{2.285}$ compound at low temperatures is shown in Fig. 1. At high temperatures a typical Debye behaviour is observed, at low temperatures the specific heat exhibits two anomalies though, at $T_{\mathrm{N}}=10.5 \mathrm{~K}$ and at $T^{*}=9.5 \mathrm{~K}$. Similarly to the specific heat data, the two anomalies are observed in temperature dependence of electrical resistivity, see inset in Fig. 2.

The least-squares fitting of a linear dependence to $C / T$ vs. $T^{2}$ data in a temperature region from $16 \mathrm{~K}$ up to $30 \mathrm{~K}$ provides a value of Sommerfeld coefficient of $\gamma=70 \mathrm{~mJ} / \mathrm{mol} \cdot \mathrm{K}^{2}$. The fitting in a low temperature region (below the ordering temperature) leads to 


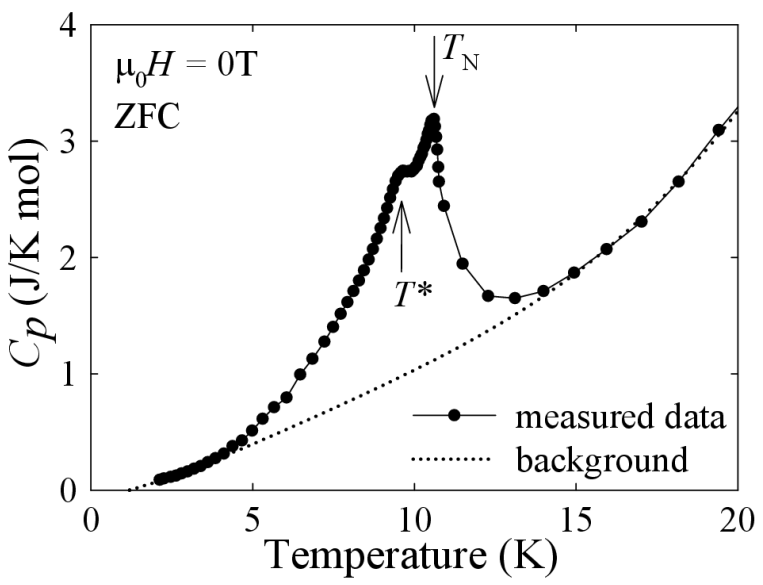

Fig. 1. Temperature dependence of specific heat in zero magnetic field at low temperatures, including an approximation of the 'background' (which is not related to the long range magnetic order).

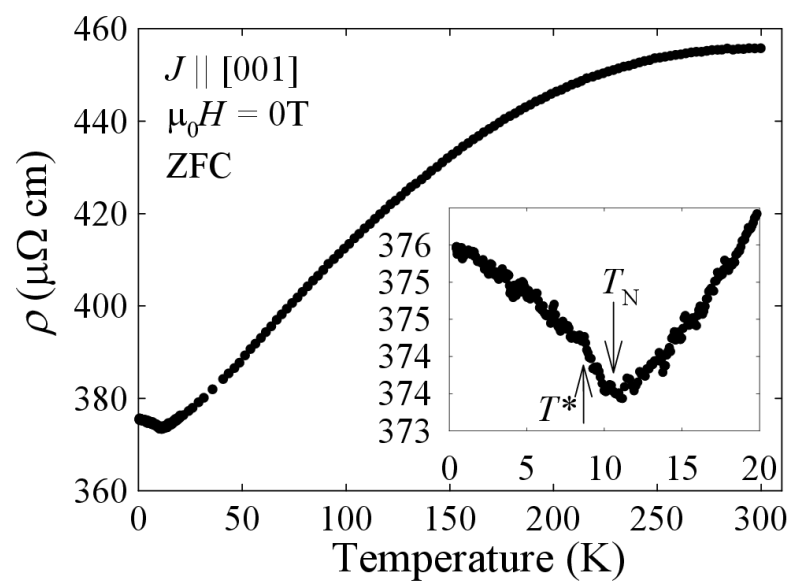

Fig. 2. Temperature dependence of electrical resistivity in zero magnetic field.

$\gamma_{\text {ord }}=28 \mathrm{~mJ} / \mathrm{mol} \cdot \mathrm{K}^{2}$. This is comparable to a similarly behaving $\mathrm{CeCoGe}_{3}$ (as concerns magnetic behaviour, see later), $\gamma_{\text {ord }}^{\mathrm{CeCGe}_{3}}=32 \mathrm{~mJ} / \mathrm{mol} \cdot \mathrm{K}^{2}[7]$.

As the non-magnetic analogue is not available for this compound, in order to estimate the magnetic entropy connected to the observed transitions we subtracted the part not related to the long range magnetic order by approximating the 'background' by a smooth curve, see Fig. 1. Via a numerical integration of the difference we were then able to estimate the change in magnetic entropy associated with magnetic ordering as $\Delta S=$ $0.78 \mathrm{~J} / \mathrm{mol} \mathrm{K}=0.14 \mathrm{R} \ln 2$. We attribute the low value of the magnetic entropy to significant reduction of the cerium magnetic moment (see later).

To determine the nature of the ordered state below $T_{\mathrm{N}}$ measurements of magnetic field dependence of magnetization were performed, the results are plotted in Fig. 3 together with field dependence of electrical resistivity at

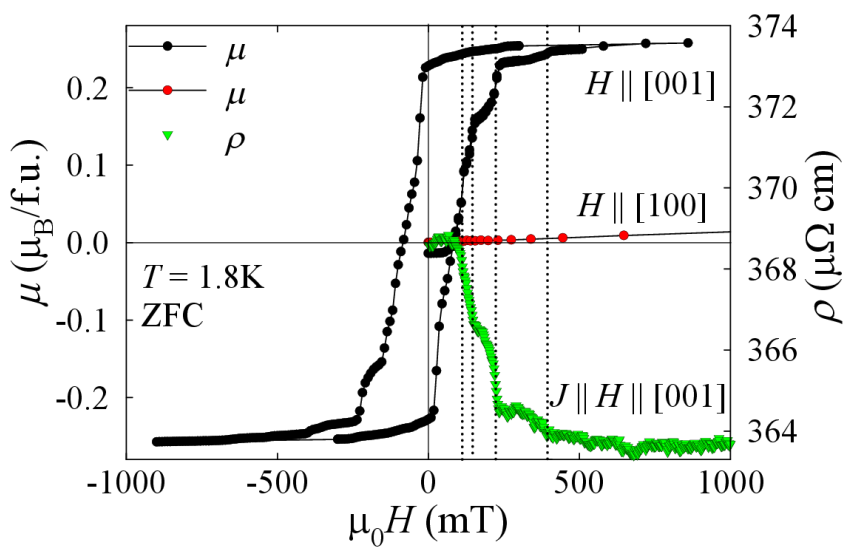

Fig. 3. Magnetic field dependence of magnetization at low temperature with field applied in both [100] and [001] direction. Dependence of electrical resistivity is shown as well.

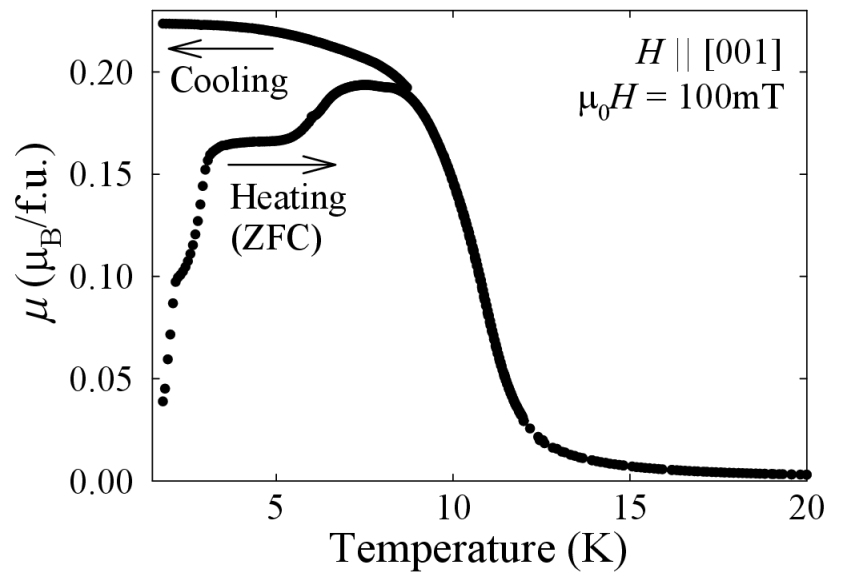

Fig. 4. Temperature dependence of magnetization in small magnetic field showing thermal hysteresis.

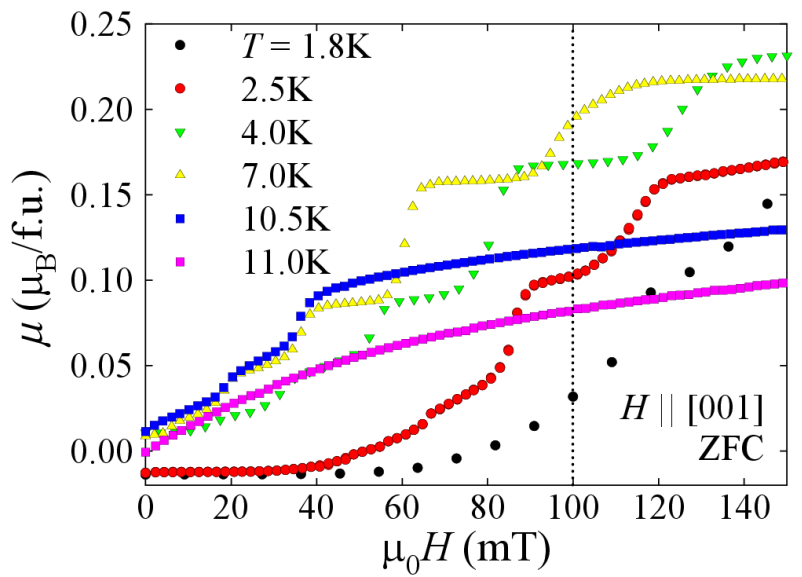

Fig. 5. Magnetic field dependence of magnetization in small fields and at low temperatures, the dotted line indicates position of the temperature scan in Fig. 4. 
the same temperature. Below $T_{\mathrm{N}}$ the compound exhibits a strong hysteresis of magnetization with respect to varying magnetic field or temperature (see Fig. 4) as well as a strong anisotropy with easy axis corresponding to the [001] direction. The magnetization is saturated above $1 \mathrm{~T}$ and stayed unchanged up to $14 \mathrm{~T}$, which was the maximum field applied within our study. The saturated value of magnetization, however, is considerably reduced $\left(0.26 \mu_{\mathrm{B}} / \mathrm{Ce}\right)$ with respect to the free $\mathrm{Ce}^{3+}$ ion value of $2.14 \mu_{\mathrm{B}}$. Besides, the compound exhibits quite interesting behaviour in small magnetic fields below $500 \mathrm{mT}$. As can be seen in Fig. 5 the compound undergoes numerous magnetic phase transitions when magnetic field is applied in $H \|[001]$ direction. Below the temperature of $11.0 \mathrm{~K}$ firstly two phase transitions appear at $34 \mathrm{mT}$ and $18 \mathrm{mT}$, further on, with decreasing temperature the transitions shift to higher magnetic fields and new transitions appear. At temperatures below $2.5 \mathrm{~K}$ the number of transitions is again reduced while still being shifted to higher magnetic fields.

The exhibited step-like behaviour of magnetization is similar to the behavior of the so-called "devil's staircase" systems (e.g. CeSb [8], $\mathrm{CeRh}_{3} \mathrm{Si}_{2}$ [9], etc.) or $\mathrm{CeCoGe}_{3}$ system [7]. As shown in Fig. 3 the numerous magnetic phase transition are also manifested in magnetic field dependence of electrical resistivity, however, the step-like behaviour is more pronounced in the magnetization data.

As shown in Fig. 6 at high temperatures $(200 \mathrm{~K} \leq$ $T \leq 350 \mathrm{~K})$ magnetic susceptibility obeys the CurieWeiss law, however, it should be stated that a small but non-negligible deviation of the data is observed at high temperatures with field $H \|[001]$. Least-squares fit of the Curie-Weiss law yields an effective magnetic moment of $\mu_{\text {eff }}^{\| c}=2.8 \mu_{\mathrm{B}} / \mathrm{Ce}$ and $\mu_{\mathrm{eff}}^{\| a}=3.97 \mu_{\mathrm{B}} / \mathrm{Ce}$ and a paramagnetic Curie-Weiss temperature of $\theta_{\mathrm{p}}^{\| c}=-276 \mathrm{~K}$ and $\theta_{\mathrm{p}}^{\| a}=-928 \mathrm{~K}$ for $H \|[001]$ and $H \|[100]$, respectively.

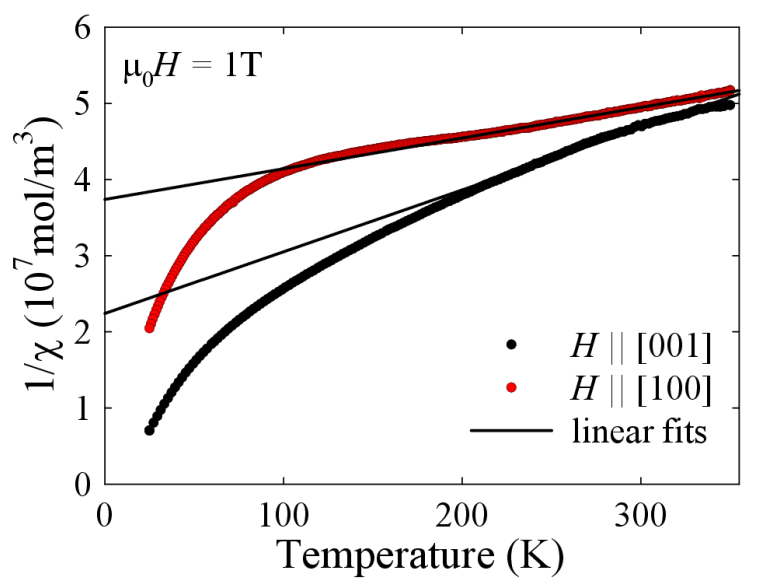

Fig. 6. Temperature dependence of magnetic susceptibility with magnetic field applied in both [100] and [001] direction. Fits of the Curie-Weiss law are shown as well.

\section{Conclusions}

Single crystal of the $\mathrm{CeCo}_{0.715} \mathrm{Si}_{2.285}$ compound has been grown by Czochralski method and characterized for the first time. Single crystal X-Ray diffraction determines that its crystal structure belongs to the spacegroup 121 ( $I-4 m 2)$ with extremely elongated unit cell $(a=4.12 \AA, c=32.84 \AA)$. The negative Curie-Weiss temperature indicates predominance of antiferromagentic interactions and reflects the anisotropy of the crystallographic structure. The compound orders at $T_{\mathrm{N}}=10.5 \mathrm{~K}$, having another order-to-order transition at $9.5 \mathrm{~K}$. The application of magnetic field along the $c$-axis in the ordered state reveals rather rich magnetic phase diagram at low fields with saturated magnetization reaching only $0.26 \mu_{\mathrm{B}} / \mathrm{Ce}$ at $7 \mathrm{~T}$, whereas with application along the $a$-axis one observes linear behaviour (with $0.1 \mu_{\mathrm{B}} / \mathrm{Ce}$ at $7 \mathrm{~T}$ ) expected for the hard axis magnetization which we attribute to the magnetism of conduction electrons.

\section{Acknowledgments}

Experiments were performed in MLTL, which is supported within the program of Czech Research Infrastructures (project no. LM2011025).

\section{References}

[1] E. Lengyel, M. Nicklas, N. Caroca-Canales, C. Geibel, Phys. Rev. B 88, 155137 (2013).

[2] M. Giovannini, M. Hadwig, R. Pasero, E. Bauer, G. Hilscher, M. Reissner, P. Rogl, H. Michor, J. Phys.: Condens. Matter 22, 135601 (2010).

[3] M. Pelizzone, H.F. Braun, J. Muller, JMMM 30, 33 (1982).

[4] M. Szlawska and D. Kaczorowski, J. Phys.: Condens. Matter 26, 016004 (2014).

[5] P. Haen, P. Lejay, B. Chevalier, B. Lloret, J. Etourneau, M. Sera, J. Less-Common Met. 110 , 321-5 (1985).

[6] D. Fort, J. Less-Common Met. 134, 45 (1987).

[7] A. Thamizhavel, T. Takeuchi, T. D Matsuda, Y. Haga, K. Sugiyama, R. Settai, Y. Ōnuki, J. Phys. Soc. Jpn. 74, 1858 (2005).

[8] J. Rossat-Mignod, J.M. Effantin, P. Burlet, T. Chattopadhyay, L.P. Regnault, H. Bartholin, C. Vettier, O. Vogt, D. Ravot, J.C. Achart, JMMM 52, 111 (1985).

[9] D. Kaczorowski, T. Komatsubara, Phys. B 403, 1362 (2008). 